\title{
Dielectric Spectroscopy of Two Concentrations of Magnetic Nanoparticles in Oil-Based Ferrofluid
}

\author{
Š. HARdoñ $\check{S}^{a, *}$, J. KÚdelČÍK ${ }^{a}$ AND M. GUTTEN ${ }^{b}$ \\ ${ }^{a}$ Department of Physics, Faculty of Electrical Engineering and Information Technology, \\ University of Žilina, 010 26, Slovakia \\ ${ }^{b}$ Department of Measurement and Applied Electrical Engineering, \\ Faculty of Electrical Engineering and Information Technology, University of Žilina, 010 26, Slovakia

\begin{abstract}
The study of the changes of dielectric parameters leads to a better understanding of polarization and relaxation phenomena in ferrofluids. The impacts of the electric field and magnetic flux density cause the changes in dielectric parameters and structural arrangement of magnetic nanoparticles in ferrofluid and this effect has been studied by using dielectric spectroscopy. The frequency dependence of $\tan (\delta)$ was measured within the frequency range from $1 \mathrm{mHz}$ to $10 \mathrm{kHz}$ and in a wide range of temperatures from $-25^{\circ} \mathrm{C}$ to $25^{\circ} \mathrm{C}$. Magnetic flux density and electric field were applied by a capacitance method. The object of our study was the ferrofluid with two different concentrations of magnetic nanoparticles in transformer oil MOL. The dielectric parameters were measured at parallel and perpendicular orientations of the magnetic flux density with value of $200 \mathrm{mT}$ to the electric field as a function of temperature. The Cole-Cole relaxation model was used for analysing measured data. From this model, basic dielectric parameters as a function of temperature were determined for different fields.
\end{abstract}

DOI: 10.12693/APhysPolA.137.961

PACS/topics: dielectric spectroscopy, ferrofluid, nanoparticles

\section{Introduction}

The area of nanotechnology is currently a very active area of research. Researchers have defined nanofluid as a colloidal mixture of solid nanoparticles (metals, oxides, carbides, nitrides, or nanotubes) with sizes in the range of 5-100 nm in base fluids like water or organic fluids. By dispersing nanoparticles in a vast diversity of fluids, the nanofluids have been prepared [1-4]. The thermal properties of nanofluids have been studied by many researchers, who confirmed that the addition of nanoparticles to base fluids greatly enhances their thermal conductivity [5]. Karthik et al. [6] showed that the primary aspect of any heat transfer fluid is to characterize its thermophysical properties, with thermal conductivity being a fundamental one. Application of nanofluids is expanding into energy conservation due to faster and efficient cooling and hence better performance in a wide variety of practical applications. Using nanofluids in heat exchangers, mainly in microcooling systems, may result in energy and cost savings [7, 8].

Oil-paper insulations are widely used in electrical power equipment, such as transformers and inductors, but due to the low cooling efficiency of insulating systems, the faults and degradations by thermal aging are created [9]. Transformer oil-based nanofluids have attracted a lot of attention because their thermal properties are well established regarding the addition of a given concentration of nanoparticles to base fluids [10-12].

\footnotetext{
*corresponding author; e-mail: hardon@fyzika.uniza.sk
}

In the absence of a magnetic field, a ferrofluid is a homogeneous suspension. When a magnetic field is applied on a ferrofluid, the structural changes take place. The fact that the magnetic nanoparticles (MNPs) in a ferrofluid are polarizable, the dielectric spectroscopy can also be used to study their dielectric parameters. The dependences of the dielectric permittivity and $\tan \delta$ of ferrofluids on applied electric and magnetic fields were studied by many authors $[13,14]$. In these fields, the processes in macroscopic surroundings of the electrode system and microscopic surroundings of magnetic particles lead to the creation of new structures (clusters). The dielectric parameters of ferrofluids are dependent on the value of the magnetic field and on the orientation of the magnetic and electric fields. This phenomenon, a magnetodielectric anisotropy, is studied by many researchers $[14,15]$.

\section{The experimental results of investigated ferrofluid}

The goal of this contribution was to study of frequency dependence of a magnetodielectric effect of ferrofluid in the range from $1 \mathrm{mHz}$ to $10 \mathrm{kHz}$ for the relative permittivity and $\tan \delta$. Ferrofluids with MNPs (iron oxide $\mathrm{FeO} \cdot \mathrm{Fe}_{2} \mathrm{O}_{3}$ ) and oleic acid as a surfactant was based on inhibited insulating transformer oil TO 40 A MOL [16]. Two ferrofluid samples with various magnetic volume fractions: $1.5 \%$ (FF1) and $2.5 \%$ (FF2) were studied in this preliminary study. The well-proven measurement method with device IDAX 350 was used in the experimental study of the effect of electric and magnetic fields on the relative permittivity and $\tan (\delta)$. The ferrofluid 
IDAX 350

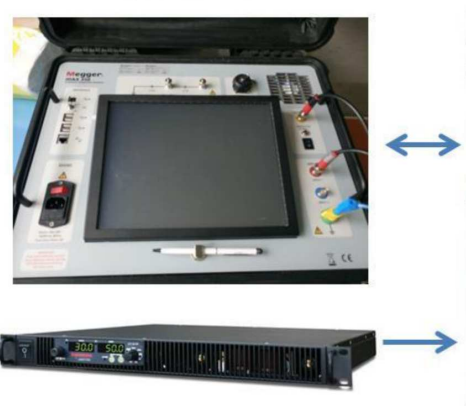

Source for electromagnet

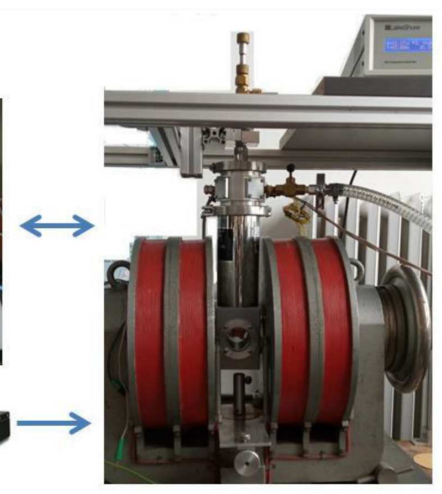

Temperature controller

LakeShore in electromagnet
Fig. 1. Experimental arrangement.

was placed in the liquid crystal cell fixed in the temperature stabilized block $\left( \pm 0.1^{\circ} \mathrm{C}\right)$ by temperature controller MODEL 325 (LakeShore) and with the ability to rotate the sample (Fig. 1). The distance between indium tin oxide electrodes in the cell was $50 \mu \mathrm{m}$ and its capacity was $C_{0}=6.5 \mathrm{pF}$. A more detailed description of the principle of measurement is presented in $[3,13]$.

The real and imaginary parts of the complex relative permittivity of the ferrofluid FF1 measured in the absence of the magnetic flux density at the constant electric field of $140 \mathrm{kV} / \mathrm{m}$ are depicted in Fig. 2a. The real permittivity increases from $10 \mathrm{~Hz}$ with decreasing frequency of the electric field. At higher frequencies, its value is constant and increases with temperature. The imaginary part of permittivity is almost linear in the log-log scale, and increases with decrease in the frequency and its value is also dependent on the temperatures. Figure $2 \mathrm{~b}$ shows the development of $\tan \delta$ as a function of the frequency at three temperatures. The local maximum at an eigenfrequency $\left(f_{e}=\frac{1}{2} \pi \tau\right)$ relates to the relaxation process of polarization. For an explanation of this maximum, the Schwarz model of electric double-layer polarization can be used [17]. The effect of the temperature dependence of the dielectric polarization causes the shifts of $\tan (\delta)$ and eigenfrequency $f_{e}$ to the higher values of frequencies $\left(-25^{\circ} \mathrm{C}-0.5 \mathrm{~Hz},-5^{\circ} \mathrm{C}-1 \mathrm{~Hz}, 25^{\circ} \mathrm{C}-4 \mathrm{~Hz}\right)$. The full lines in Fig. 2 and Fig. 3 represent the Cole-Cole fits of experimental data. The Cole-Cole equation is a relaxation model that is often used to describe dielectric relaxations and it has the following form [18]:

$$
\varepsilon^{*}=\varepsilon_{\infty}+\frac{\Delta \varepsilon}{1+\left(j, \omega \tau_{0}\right)^{(1-\alpha)}}+\frac{\sigma}{\varepsilon_{0} \omega},
$$

where $\varepsilon^{*}$ is the complex dielectric constant, $\varepsilon_{\infty}$ is the "infinite frequency" dielectric constants, $\omega$ is the angular frequency, $\tau$ is a time constant of the relaxation process, and $\sigma$ is the DC conductivity. The distribution parameter $\alpha$, which takes a value between 0 and 1 , allows describing different spectral shapes. For the fit of experimental data Origin 7.0 software was used. Obtained parameters from the fit by the Cole-Cole equation are shown in Table I.
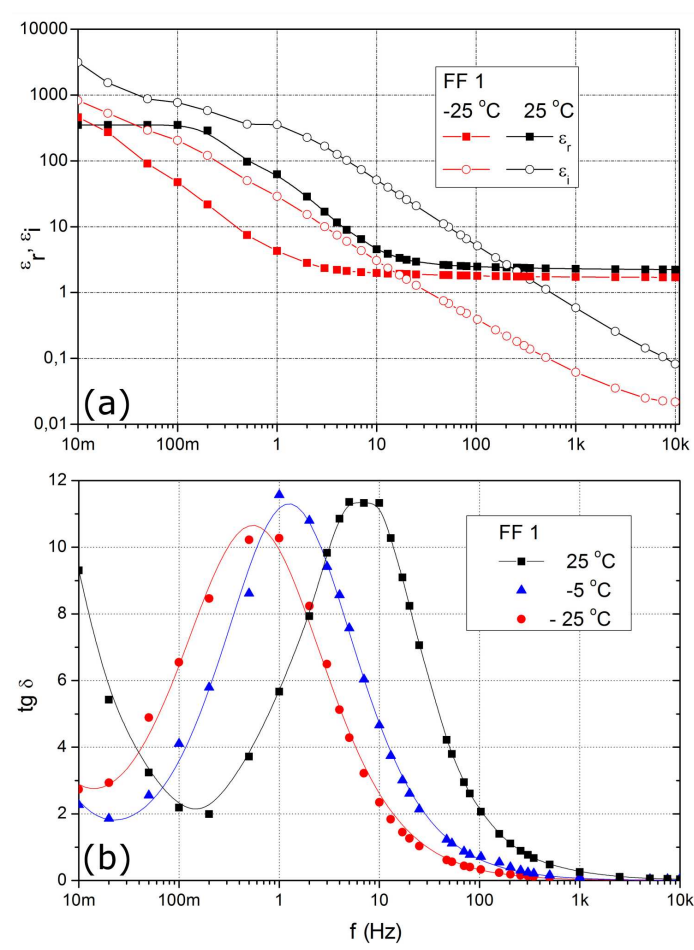

Fig. 2. (a) The dependence of the real and imaginary permittivity on the frequency at temperatures $-25^{\circ} \mathrm{C}$ and $25^{\circ} \mathrm{C}$. (b) The dependence of $\tan \delta$ on the frequency of the electric field at three temperatures.
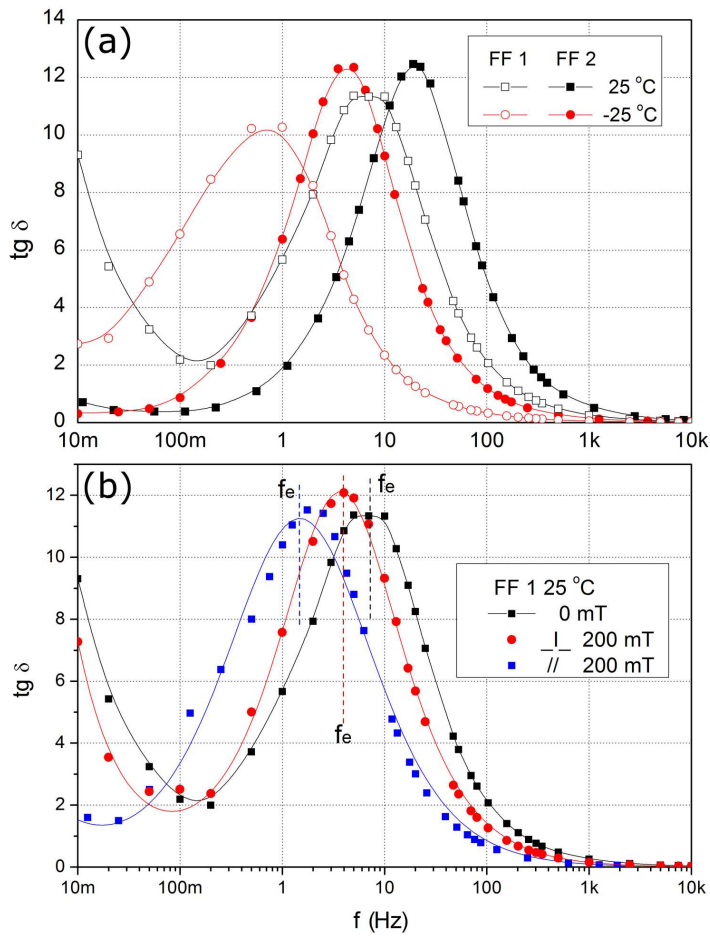

Fig. 3. Dependence of the $\tan \delta$ on the frequency at constant electric field $140 \mathrm{kV} / \mathrm{m}$ : (a) FF1 and FF2 at temperatures $-25^{\circ} \mathrm{C}$ and $25^{\circ} \mathrm{C}$ at $B=200 \mathrm{mT}$, and (b) $\mathrm{FF} 1$ at $25^{\circ} \mathrm{C}$ without and at the magnetic flux density $B=200 \mathrm{mT}$ for two different orientations of electric and magnetic fields. 


\section{TABLE I}

The calculated values for $\tan \delta$ by the Cole-Cole relaxation model $[3,18]$.

\begin{tabular}{l|c|c|c|c|c|c}
\hline \hline Temp. & \multicolumn{3}{|c|}{$-25^{\circ} \mathrm{C}$} & \multicolumn{3}{c}{$25^{\circ} \mathrm{C}$} \\
\hline$B[\mathrm{mT}]$ & 0 & $\| 200$ & $\perp 200$ & 0 & $\| 200$ & $\perp 200$ \\
\hline \multicolumn{7}{c}{$\mathrm{FF} 1$} \\
\hline$\tau[\mathrm{s}]$ & 12.46 & 38.30 & 21.6 & 0.30 & 4.87 & 0.90 \\
$f_{e}[\mathrm{~Hz}]$ & 0.55 & 0.12 & 0.34 & 6.30 & 1.54 & 3.70 \\
$\alpha$ & 0.84 & 0.92 & 0.90 & 0.96 & 0.95 & 1.00 \\
$\sigma[\mathrm{nS}]$ & 0.28 & 0.08 & 0.12 & 1.70 & 0.26 & 1.40 \\
\hline \multicolumn{7}{c}{$\mathrm{FF} 2$} \\
\hline$\tau[\mathrm{s}]$ & 0.90 & 0.90 & 0.91 & 0.41 & 0.70 & 0.58 \\
$f_{e}[\mathrm{~Hz}]$ & 4.00 & 1.85 & 0.70 & 20.0 & 12.00 & 14.80 \\
$\alpha$ & 0.90 & 0.90 & 0.91 & 0.86 & 0.88 & 0.86 \\
$\sigma[\mathrm{nS}]$ & 0.22 & 0.03 & 0.09 & 1.10 & 0.67 & 0.85
\end{tabular}

Comparison of the dependence of $\tan \delta$ of studied ferrofluids as a function of the frequency of the electric field without application of the magnetic flux density at two temperatures is shown in Fig. 3a. These developments have the local maximum, position $\left(f_{e}\right)$ of which depends on the temperature and concentration of MNPs (Table I). This relaxation maximum is connected with electric double-layer (EDL) around each nanoparticle in an electric field [13]. The development of $\tan \delta$ shifts to higher frequencies with increasing concentration of magnetic nanoparticles.

Figure 3b shows the magneto-dielectric effect of $\tan \delta$ of FF1 at the various orientations of the magnetic flux density and the electric field. The magnetic flux density caused a shift in the whole development of $\tan \delta$ to lower frequencies. This shift was caused by a rearrangement of MNPs to new structures in the presence of magnetic flux density. In these structures, the EDL of each nanoparticle at the contact regions was changed because the counter ions were moved by the electric field on the surface of these structures, and so it looked like a particle with a bigger radius $[9,18]$. The bigger radius of structures is connected with the increase of the relaxation time $\tau$ and decrease of eigenfrequency $f_{e}$ (Table I). From the Cole-Cole fit it can also be seen that the conductivity $(\sigma)$ of ferrofluids in the magnetic field decreases. This decrease is connected with the presence of bigger nanoparticle structures, which capture more electric charges. The decrease of $\tan \delta$ was more visible for the parallel orientation of these fields than for perpendicular orientation. At the perpendicular orientation of fields, the Lorentz force has a maximum effect on the drift movement of nanoparticles. This causes them move in a spiral, which causes longer tracks of the nanoparticles in the system and it is connected with the increase of $\tan \delta$. The same effect of the shift of development of $\tan \delta$ to lower frequencies with the application of the magnetic flux density was observed for lower temperatures and ferrofluid FF2 (see Table I).

\section{Conclusions}

Using the dielectric spectroscopy the influence of the magnetic flux density on the structural rearrangement of MNPs in ferrofluid was studied. The development of $\tan (\delta)$ of studied ferrofluid showed one low-frequency relaxation maximum at the eigenfrequency which was associated with the electric double-layer. The magnetodielectric effect of $\tan (\delta)$ was observed in ferrofluids subjected to the parallel and perpendicular orientation of the magnetic to the electric field. When the magnetic flux density was applied, the whole development of $\tan \delta$ shifted to the left side and this shift resulted from a rearrangement of nanoparticles to new structures.

\section{Acknowledgments}

The work was supported by Slovak Grant Agency VEGA $1 / 0510 / 17$.

\section{References}

[1] V. Segal, K. Raj, Ind. J. Eng. Mater. Sci. 5, 416 (1998).

[2] A. Kumar, S. Subudhi, Heat Mass Transf. 54, 241 (2017).

[3] Š. Hardoň, J. Kúdelčík, E. Jahoda, M. Kúdelčíková, Int. J. Thermophys. 40, 24 (2019).

[4] A. Hidayat, W. Elyani, A. Puspitaningrum, IOP Conf. Series Mater. Sci. Eng. 515, 012092 (2019).

[5] S. Tagamouti, S.E. Bouzit, L.C. Costa, M.P.F. Graca, A. Outzourhit, Spectrosc. Lett. 45, 761 (2015).

[6] R. Karthik, R. Harish Nagarajan, B. Raja, P. Damodharan, Exp. Therm. Fluid Sci. 40, 1 (2012).

[7] J.A. Eastman, S.U.S. Choi, S. Li, W. Yu, L.J. Thompson, Appl. Phys. Lett. 78, 718 (2000).

[8] Z.L. Wang, D.W. Tang, S. Liu, X.H. Zheng, N. Araki, Int. J. Thermophys. 28, 1255 (2007).

[9] J. Miao, D. Ming, R. Ming, W. Xuezhou, S. Liangping, W. Hao, J. Appl. Phys. 113, 0021 (2013).

[10] V. Mentlik, P. Trnka, J. Hornak, P. Totzauer, Energies 11, 508 (2018).

[11] A.V. Primo, B. Garcia, A. Ricardo, IEEE Electr. Insulat. Magn. 34, 3 (2018).

[12] J. Ghasemi, S. Jafarmadar, M. Nazari, J. Magn. Magn. Mater. 389, 148 (2015).

[13] S. Hardon, J. Kudelcik, P. Bury, M. Gutten, Acta Phys. Pol. A 113, 477 (2018).

[14] M. Timko, K. Marton, L. Tomco, et al., Magnetohydrodynamics 48, 427 (2012).

[15] P. Zukowski, T.N. Koltunowicz, V. Bondariev, A.K. Fedotov, J.A. Fedotova, J. Alloys Compd. 683, 62 (2016).

[16] S. Hardon, J. Kudelcik, M. Rajnak, AIP 1996, 020016 (2018).

[17] I. Malaescu, C.N. Marin, J. Coll. Interf. Sci. 251, 73 (2002).

[18] K.S. Cole, R.H. Cole, J. Chem. Phys. 9, 341 (1941). 